Research Article

\title{
Fault Diagnosis of Rotating Machinery Based on Stochastic Resonance with a Bistable Confining Potential
}

\author{
Zhixing Li $\mathbb{D i D}^{1,2}$ and Boqiang Shi $\mathbb{D}^{1}$ \\ ${ }^{1}$ School of Mechanical Engineering, University of Science \& Technology Beijing, Beijing, China \\ ${ }^{2}$ School of Mechanical Engineering, Inner Mongolia University of Science \& Technology, Baotou, China \\ Correspondence should be addressed to Boqiang Shi; shiboqiang@ustb.edu.cn
}

Received 18 May 2018; Revised 1 September 2018; Accepted 3 September 2018; Published 17 October 2018

Academic Editor: Daniele Baraldi

Copyright ( $) 2018$ Zhixing Li and Boqiang Shi. This is an open access article distributed under the Creative Commons Attribution License, which permits unrestricted use, distribution, and reproduction in any medium, provided the original work is properly cited.

\begin{abstract}
Since the weak fault characteristics of mechanical equipment are often difficult to extract in strong background noise, stochastic resonance (SR) is widely used to extract the weak fault characteristics, which is able to utilize the noise to amplify weak fault characteristics. Although classical bistable stochastic resonance (CBSR) can enhance the weak characteristics by adjusting the parameters of potential model, when potential barrier height is adjusted potential well width is also changed and vice versa. The simultaneous change of both potential well width and barrier height is difficult to obtain a suitable potential model for better weak fault characteristic extraction and further fault diagnosis of machinery. For this reason, the output signal-to-noise ratio (SNR) of CBSR is greatly reduced, and the corresponding enhancement ability of weak fault characteristics is limited. In order to avoid the shortcomings, a new SR method is proposed to extract weak fault characteristics and further diagnose the faults of rotating machinery, where the classical bistable potential is replaced with a bistable confining potential to get the optimal SR. The bistable confining potential model not only has the characteristics of the classical bistable potential model but also has the ability to adjust the potential width, barrier height, and wall steepness independently. Simulated data are used to demonstrate the proposed new SR method. The results indicate that the weak fault characteristics can be effectively extracted from simulated signals with heavy noise. Experiments on the bearings and planetary gearboxes demonstrate that the proposed SR method can correctly diagnose the faults of rotating machinery and moreover has higher spectrum peak and better recognition degree compared with the CBSR method.
\end{abstract}

\section{Introduction}

The vibration test is often used method in fault diagnosis of mechanical equipment, but the mechanical equipment is usually affected by heavy noise, multi vibration source excitation and response mutual coupling, human disturbance, and so on [1-3]. All interferences cause the signal-to-noise ratio (SNR) to be very small, and the fault characteristic frequency is difficult to extract. In addition, the early fault characteristics are fainter in the bad outside environment. Therefore, the weak fault characteristics extraction is a key problem in mechanical fault diagnosis $[4,5]$. The commonly used fault diagnosis methods contain wavelet transform [6], ensemble empirical mode decomposition [7-9], local mean decomposition [10,11], singular value decomposition [12], and so on $[13,14]$. The above methods show good performance in fault diagnosis and weak fault characteristics extraction, but they detect fault characteristics from the view of eliminating noise. For the weak fault characteristics of heavy noise pollution, noise reduction is effective, but the weak fault characteristic frequency is weakened. Therefore, the traditional signal processing methods cannot effectively extract the weak fault characteristics which are polluted by the heavy noise. However, stochastic resonance (SR) is the theory using noise to enhance hidden signal [15]. SR was proposed by Benzi et al. in 1981 [16]. At present, SR has been widely used in fault diagnosis. For the superiority of the SR in the extraction of weak fault characteristics, SR has gradually become the focus of research in signal processing. At present, a large number of scholars have studied the weak 
signal detection technology in strong background noise. According to the approximate adiabatic theory, SR is only applicable to the condition of small parameters [17]. Leng et al. [18] proposed two sampling frequency conversion in order to achieve the large parameter signals SR. Tan et al. [19] used frequency-shifted and rescaling detection technology, which overcame the limitation of the small parameter condition of the traditional SR for detecting high frequency signals in practical engineering. Lai and Leng [20] proposed linear amplitude transformation, time frequency scaling, and parameter tuning methods for signal detection in large frequency and high intensity noise. These studies provide effective methods for the detection of large parameter signal with SR. The classical bistable stochastic resonance (CBSR) is the most commonly used method to detect the weak fault characteristics. Many scholars have done a lot of research on CBSR method. Lei et al. [21] proposed an underdamped SR method with stable-state matching to achieve the best matching between stable-state types and input signals and applied to fault characteristic frequency extraction of rolling element bearings. $\mathrm{Lu}$ et al. [22] proposed a full wave signal to make better the construction of SR and applied to fault characteristic frequency extraction of the wear bearing, Liu et al. [23] proposed improved artificial fish-swarm algorithm for parameters matching to achieve optimal SR. Zheng et al. [24] proposed energy harvesting experiment which is applied to SR. Ding et al. [25] proposed a method of fusion characteristics extraction for local reservation prediction in order to find out the difference between the bearing state and the health condition. Han et al. [26] proposed a weak signal extraction method for multistable SR of wavelet transform and parameter compensation bandpass which is used to detect multifrequency signals. Shi et al. [27] proposed asymmetric bistable SR driven by unrelated multiplication and additive noise. Li et al. [28] proposed the SNR of the principal component and residual in the SR to realize quantitative analysis of SR. Duan et al. [29] proposed a new form of SR based on coding efficiency measurement. Li and Shi $[30,31]$ proposed the SR extraction method for adaptive singular value decomposition. In addition, a stochastic resonance potential model based on chaotic ant colony algorithm is proposed and used in fault diagnosis of planetary gearbox. These studies are based on the simultaneous adjustment of barrier height and potential width. As long as the potential barrier height changes, the potential width is also changed and vice versa. In this case, the perfect potential model structure cannot be obtained. Therefore, many scholars have substituted the potential model of CBSR with other potential models to expect better SR. Lu et al. [32] proposed monostable and tristable SR and applied them on bearing experiments, and the results show that the two methods are superior to the CBSR method in enhancing the weak fault characteristics. Qiao et al. [33] proposed the piecewise mixed $\mathrm{SR}$, and the new potential model is applied to fault diagnosis experiment of the planetary gearbox and the method has achieved good results. However, all of the above methods change the structure of classical bistable potential model. When the potential barrier height is adjusted, the potential width is changed and vice versa and do not fundamentally solve the problem of structural adjustment of classical bistable potential model. The potential width and barrier height which change simultaneously are difficult to obtain perfect potential model structure. This shortcoming makes to obtain the target characteristic frequency difficulty and limits the enhancement ability of weak fault characteristics in CBSR method. Therefore, how to adjust the structure of the potential model better is the key problem to achieve the best SR. In order to avoid the problems of the CBSR method, this paper proposed an adaptive bistable confining potential stochastic resonance (BCPSR) method, which establishes the bistable confining potential model to make the potential width, barrier height, and wall steepness adjusted independently and simultaneously, so the potential model can better match parameters and SR.

In Section 2, the bistable confining potential model and strategy of the BCPSR method for weak signal extraction are introduced. In Section 3, the validity of simulation signal is verified by the new method comparing with the CBSR method. In Section 4, the new method was applied to the fault characteristic signal extraction of the bearing and the planetary gearbox. The diagnostic results reveal that the new method can identify mechanical failure. Compared to the CBSR method, the new method has the higher characteristic frequency spectrum peak and recognition degree. The conclusions are drawn in Section 5.

\section{SR Model with Bistable Confining Potential}

2.1. Theoretical Model. Most of the research studies on SR focus on the traditional bistable model. The CBSR can be explained as a particle driven by noises and the cycle force. As the particles are driven by noise, the cycle force will be enhanced. The governing equation that illustrates such a phenomenon under the assumption of overdamped condition can be introduced by the following equation:

$$
\frac{d x}{d t}=-U^{\prime}(x)+A \sin \left(2 \pi f_{\mathrm{d}} t+\varphi\right)+N(t)
$$

where $A$ is the amplitude of the cycle signal, $f_{\mathrm{d}}$ is the driving frequency, and $\varphi$ is phase. Meanwhile, the noise item $N(t)$ needs to satisfy the following conditions:

$$
\begin{aligned}
N(t) & =\sqrt{2 D} \xi(t), \\
\langle N(t), N(t+\tau)\rangle & =2 D \delta(t),
\end{aligned}
$$

in which $D$ is the strength of the noise and $\xi(t)$ represents an added Gaussian white noise (AGWN) where the mean value is 0 and the variance is 1 . For the classical bistable potential model, $U(x)=U_{b}(x)$ represents a reflection-symmetric quartic potential:

$$
U_{b}(x)=-\frac{a}{2} x^{2}+\frac{b}{4} x^{4},
$$

in which $a$ and $b$ denote the potential parameters of the classical bistable model. $x(t)$ denotes the SR system output, and Equations (1) and (3) describe essentially the overdamped motion of the particle driven by external force 
signal (periodic signal) and noise in double potential wells. The potential function $U_{b}(x)$ has two fixed positions at $x= \pm \sqrt{a / b}$, the barrier altitude is $\Delta U=a^{2} / 4 b$, and the highest point of barrier is located at $x=0$, and these characteristics are shown in Figure 1. It is similar for the bistable confining potential model, $U(x)=U_{\mathrm{cb}}(x)$, which is a symmetric nonlinear potential and is shown as follows:

$$
U_{\mathrm{cb}}(x)=U_{0} \exp \left(\frac{-x^{2}}{L_{0}^{2}}\right)+k \frac{|x|^{q}}{q}
$$

in which the $k$ is the remaining tunable parameter with positive real value in bistable confining potential. In order to ensure the constraints, we ask for $q>1$. The whole potential model is symmetrical at $x=0$, i.e., $U_{\mathrm{cb}}(x)=U_{\mathrm{cb}}(-x)$.

To analyze the difference and similarity between the classical bistable potential and the bistable confining potential, the bistable potential curves with different potential parameters are described in Figure 1. It is clear that the height and width of potential can be controlled by the parameters $a$ and $b$. In other words, under the same $b$ condition, the greater the $a$ is, the higher the potential barrier is; under the same $a$ condition, the greater the $b$ is, the lower the potential barrier is. This phenomenon can satisfy the theoretical value $\Delta U_{b}=a^{2} /(4 b)$. Meanwhile, the bistable confining potential curves with different parameters can also be shown in Figure 2. It is clear that the different potential structure can be caused by adjusting the potential parameters. From Figure 2(a), under any other parameters unchanged, we can see the bistable confining potential also has two wells and one barrier, which is similar to the classical bistable potential. In addition, with the increase of parameter $q$, the barrier height of potential well has no change, but the minimum value points slowly move the middle point $x=0$. It can cause greater wall steepness of the potential and smaller width of potential. In other words, the parameter $q$ can determine the wall steepness and width of potential. Similarly, we can fix any other parameters to observe the change of the potential with one parameter. In Figure 2(b), it can be found that the smaller the $L_{0}$ is, the higher the barrier height of potential is. Likewise, with the increase of $L_{0}$, the potential width becomes greater. In Figures 2(c) and 2(d), they can see that the greater the $U_{0}$ is, the higher the barrier height of potential, and the greater the $k$ is, the smaller the barrier height and the greater wall steepness of potential and vice versa. Through the above analysis, we can see that the two models have similar adjustment characteristics through adjusting the potential parameters. Additionally, the two potential models are all bistable potential which has one barrier and two symmetric potential wells. However, the difference between the two models is that the wider range of wall steepness and potential width can be obtained by tuning the parameters of bistable confining potential, and likewise more detailed potential structures can be realized completely.

Substitute Equation (4) to Equation (1), the SR based on the bistable confining potential can be obtained as follows:

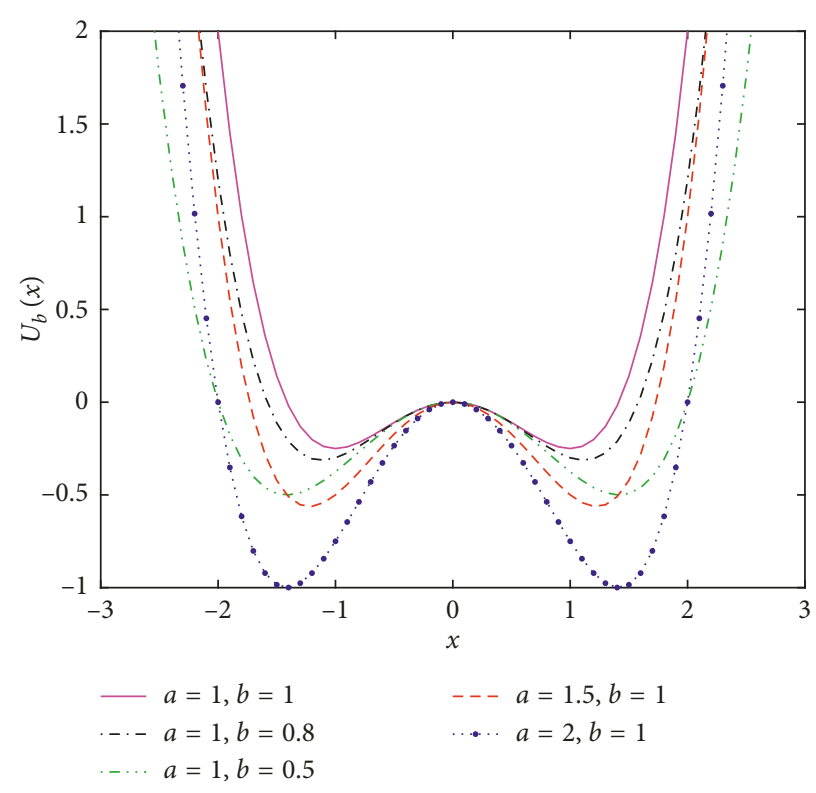

FIgURE 1: The bistable potential function with different parameters.

$$
\begin{aligned}
\frac{d x}{d t}= & \frac{2 U_{0}}{L_{0}^{2}} x \exp \left(\frac{-x^{2}}{L_{0}^{2}}\right)-k \operatorname{sgn}(x)|x|^{q-1} \\
& +A_{0} \sin \left(2 \pi f_{0} t+\varphi\right)+n(t) .
\end{aligned}
$$

The $\operatorname{sgn}(x)$ is the symbol function. It can be understood from Equation (5) that the system can be adjusted by parameters $U_{0}, L_{0}, q$ and $k$ to enhance the output signal. Equation (5) can be calculated using the discrete fourth-rank Runge-Kutta method as shown below:

$$
\left\{\begin{array}{l}
x^{\prime}=f(t, x), x(t[0])=x[0] \\
x[n+1]=x[n]+\frac{h}{6}\left(k_{1}+2 k_{2}+2 k_{3}+k_{4}\right) \\
k_{1}=f(t[n], x[n]) \\
k_{2}=f\left(t[n]+\frac{h}{2}, x[n]+\frac{h}{2} k_{1}\right) \\
k_{3}=f\left(t[n]+\frac{h}{2}, x[n]+\frac{h}{2} k_{2}\right) \\
k_{4}=f\left(t[n]+h, x[n]+h k_{3}\right)
\end{array}\right\},
$$

in which $f(t, x)$ is a function corresponding to the right side of Equation (5) and $h$ is the calculated interval.

2.2. Weak Signal Detection Strategy Based on SR with Bistable Confining Potential. The essential of SR phenomenon is that the particles move in the potential well under the interaction of potential force, periodic force, and noise to consequently 


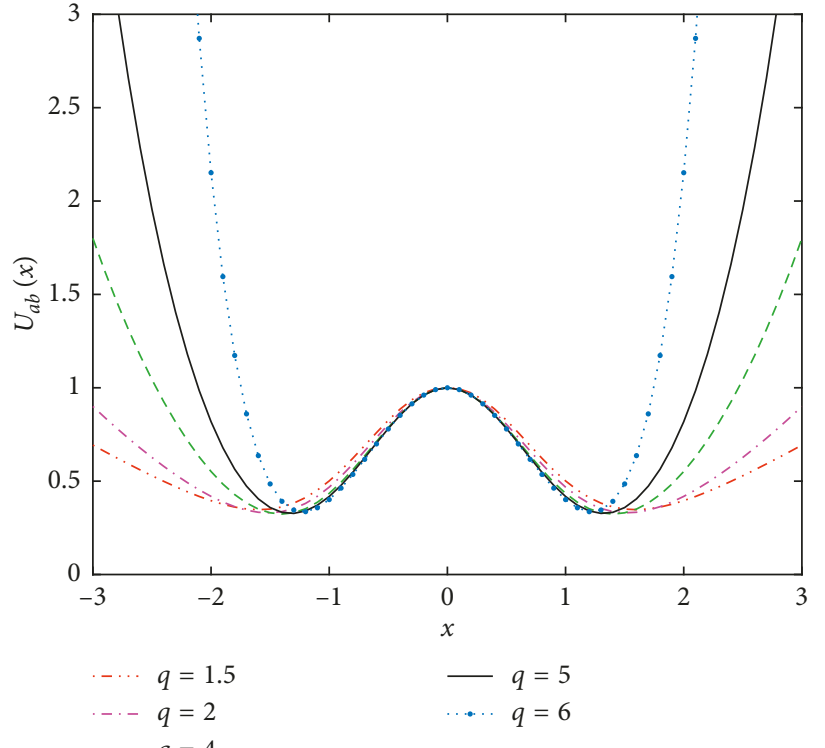

(a)

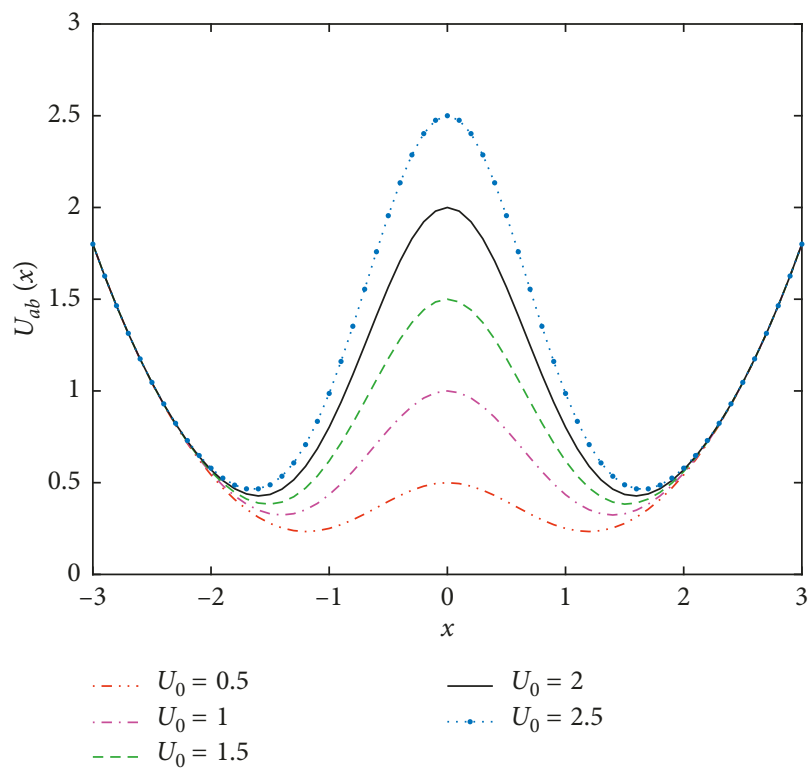

(c)

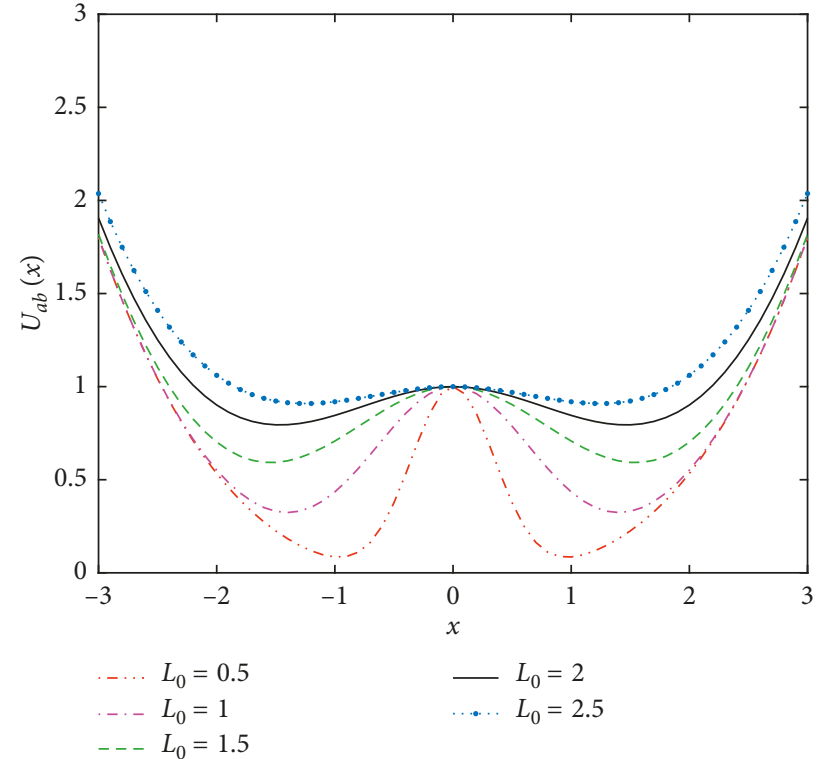

(b)

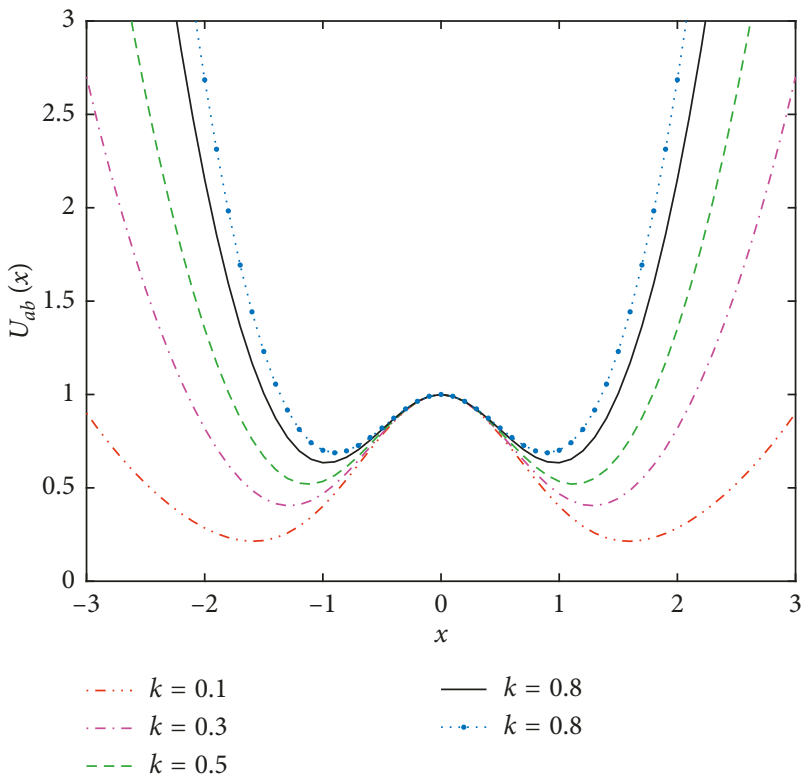

(d)

FIgURE 2: The bistable confining potential function with different parameters: (a) the bistable confining potential change with parameter $q$ at $L_{0}=1, U_{0}=1$, and $k=0.2$; (b) the bistable confining potential change with parameter $L_{0}$ at $q=3, U_{0}=1$, and $k=0.2$; (c) the bistable confining potential change with parameter $U_{0}$ at $q=3, k=0.2$, and $L_{0}=1$; and (d) the bistable confining potential change with parameter $k$ at $q=3, L_{0}=1$, and $U_{0}=1$.

achieve the enhancement of system output. In three forces, the periodic force and the noise are fixed. Thus, the effect of SR depends on the change in potential force. In general, if the distance between two potential walls is too far, the particles could not arrive to the wall edge that it is must give the greater reverse elasticity. On the other hand, when the distance between two potential walls is too narrow, the particles cannot arrive to the potential wall edge, so it will go back prematurely. Similarly, if the potential wall is too steep, the particles may rebound rapidly due to an intense reverse elasticity. When the restoring speed is too fast, the periodic oscillation could not catch up with the restoring speed. If the wall is too gentle, it cannot give a large enough acceleration to cause the particles to periodic oscillation. Therefore, when the potential model has the optimal structure, the system output can have the best enhancement effect.

With these features, to enhance the periodic signal to an extreme, the potential model should be well adjusted. For the classical bistable potential (Equation (3)), it is well known that the potential features can be determined jointly by the parameters $a$ and $b$. In other words, it is impossible to tune one isolate potential feature (e.g., potential width) while 
keeping the others (e.g., potential wall steepness) invariable by adjusting the parameters. In this case, we might assume that the potential wall steepness has been adjusted to the optimal state, but the optimal barrier height and wall steepness may not be in the best condition. Hence, the periodic signal cannot be enhanced optimally. However, we can look back to the bistable confining potential model as exhibited in Figure 2, and it can be found that the parameters $q$, $L_{0}$, and $U_{0}$ can mainly determine the potential wall steepness and barrier height. Additionally, the parameter $k$ can mainly determine the potential width, barrier height, and wall steepness. It is sure that the bistable confining potential can achieve the better potential features than classical bistable. For example, keeping the optimal potential width by tuning parameter $k$, and then the relative optimal wall steepness and barrier height can be obtained, respectively, by adjusting the parameters $q, L_{0}$, and $U_{0}$.

To optimally extract the target signal from the strong noise, the optimal input signal can be obtained by tuning the potential parameters. Here, the performance of the weak signal detection is evaluated by employing the output SNR as a criterion. Subsequently, a strategy to extract the weak fault characteristics signal based on SR with bistable confining potential is synthetically presented as shown in Figure 3 . The flow chart is described as follows:

(1) Signal preprocessing: some common technologies include filtering part of noises, detecting the driving frequency by signal demodulation when the original signal is modulated, and the shifting-frequency rescaling transform which can be performed to satisfy the small parameter condition when the driving frequency is greater than 1 .

(2) Parameter initialization: initialize the computed ranges of the bistable confining potential parameters $q, L_{0}, U_{0}$, and $k$.

(3) Output calculation: compute the output waveform by using Equation (6), and then solve the frequency spectrum and get the SNR.

(4) Output assessment: look for the maximum SNR in ternary array that corresponds to the varying variables $q, L_{0}, U_{0}$, and $k$ by using ant colony search method and then get the best combination of parameters corresponding to the maximum SNR.

(5) Postprocessing of the processed signal: the processed signal is input to the SR system to calculate the final output and realize the fault diagnosis of the mechanical equipment.

\section{Performance Evaluation}

3.1. Effect of the SR with Bistable Confining Potential. To intuitively illustrate the generated interaction between the system output and the potential parameters, a simulated failure bearing signal is produced and processed by the BCPSR method as mentioned above. As the rolling bearing is often in the form of impact, the periodic unilateral attenuation pulse signal with AGWN is selected as the simulated

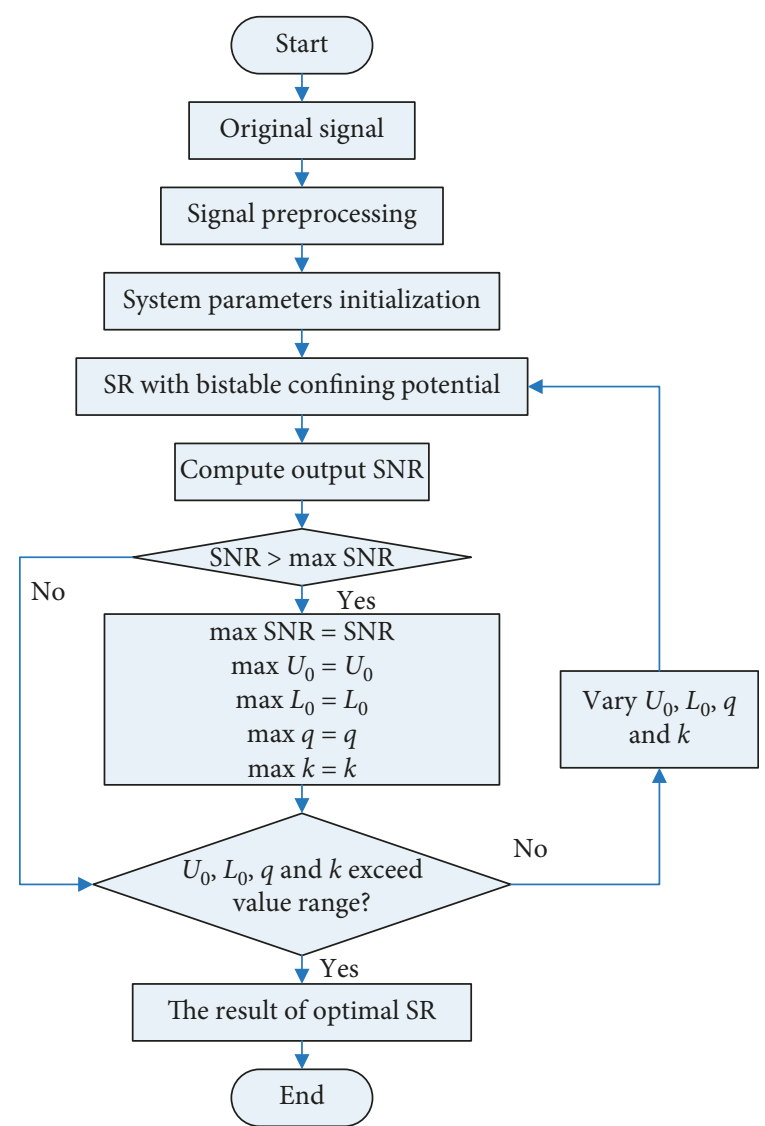

FIGURE 3: Proposed strategy to detect weak signal based on SR with bistable confining potential.

signal waveform. The simulated waveform is generated by virtue of the below equation:

$$
s(t)=A \sin (2 \pi f t) \cdot \exp \left\{-d\left[t-n(t) T_{\mathrm{d}}\right]^{2}\right\}+\sqrt{2 D} \xi(t),
$$

where $A=1$ is the signal amplitude, $f=1 \mathrm{kHz}$ is the modulation frequency, $d=6 f_{\mathrm{s}}\left(f_{\mathrm{s}}=10 \mathrm{kHz}\right.$ is the sampling frequency) shows the decay rate, $n(t)=\left[t / T_{\mathrm{d}}\right]$ dominates the pulses appear cyclical, $\xi(t)$ is the AGWN where the mean value is 0 and the variance is $1, \sqrt{2 D}=1$ is the noise intensity, and $T_{\mathrm{d}}=0.02 \mathrm{~s}$ is the interval where the impulse appears $\left(f_{\mathrm{d}}=1 / T_{\mathrm{d}}=50 \mathrm{~Hz}\right.$ is the driving frequency). The sampling time is $0.2 \mathrm{~s}$. The simulated bearing fault signal without the AGWN, with the AGWN, and corresponding spectrum are exhibited in Figures 4(a)-4(c), respectively.

After that, the SR equation with the bistable confining potential for dealing with the simulated bearing failure signal is as follows:

$$
\begin{aligned}
\frac{d x}{d t}= & \frac{-2 U_{0}}{L_{0}^{2}} x \exp \left(\frac{-x^{2}}{L_{0}^{2}}\right)+k \operatorname{sgn}(x)|x|^{q-1} \\
& +A \sin (2 \pi f t) \cdot \exp \left\{-d\left[t-n(t) T_{\mathrm{d}}\right]^{2}\right\}+\sqrt{2 D} \xi(t) .
\end{aligned}
$$

Since the simulated bearing fault signal is modulated, the Hilbert transform (HT) can be employed to detect the 


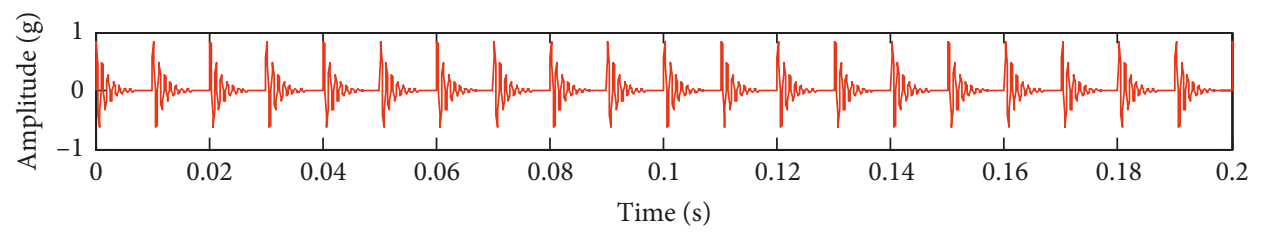

(a)

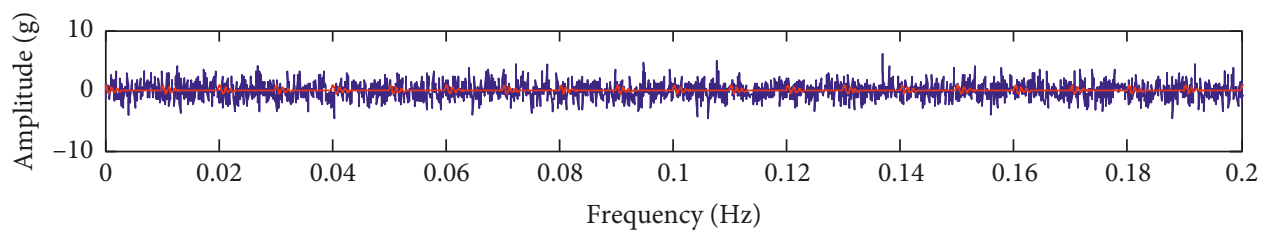

(b)

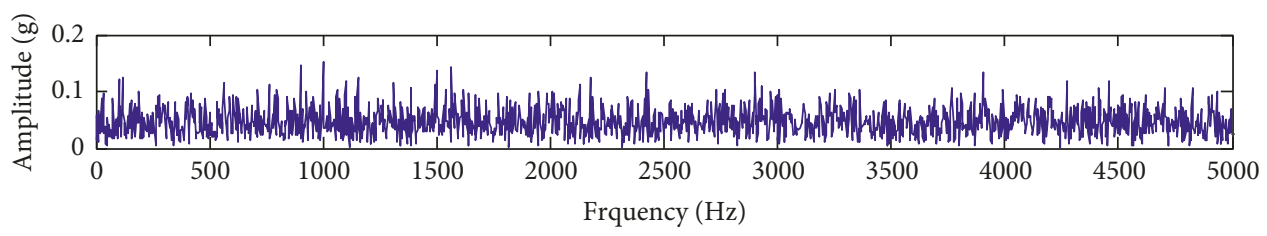

(c)

FIGURE 4: Simulated bearing fault signal: (a) periodic impulse signal without the AGWN, (b) periodic impulse signal with the AGWN, and (c) corresponding spectrum.

distinct fault-induced impulse features. Meanwhile, the envelop signal of the simulated failure bearing obtained by using HT is depicted in Figure 5(a) and its spectrum is displayed in Figure 5(b). From above figures, we can see the driving frequency is $f_{\mathrm{d}}=50 \mathrm{~Hz}$. But there are many interfering frequencies in the spectrum of envelop signal. In practical application, it is very difficult for us to identify the driving frequency of bearing fault signal, thus only depending on envelop signal the fault frequency cannot be extracted and identified exactly. In order to meet the requirement of small parameters, the envelope signals are processed by the shiftingfrequency rescaling transform method, then the parameters of bistable confining potential are optimized by ant colony algorithm. Finally, the output SNR of BCPSR method is $22.63 \mathrm{~dB}$. The time-domain waveform and spectrum are displayed in Figure 6. In Figure 6(b), it can be visualized that the characteristic frequency of the noise-contaminated signal is displayed conspicuously. The characteristic frequency spectrum peak is 0.276 , which is 0.23868 higher than that of the surrounding noise. In the interest of showing the superiority of the BCPSR method, the same signal is processed by the CBSR method. The output SNR is $20.17 \mathrm{~dB}$, which is $2.46 \mathrm{~dB}$ lower than the BCPSR method, and the time-domain waveform and spectrum are shown in Figures 6(c) and 6(d). As observed in Figure 6(d), The characteristic frequency spectrum peak is 0.1628 , which is 0.11869 higher than that of the surrounding noise. From the above analysis, it can be known that the BCPSR method has the effect of enhancing the weak fault signal obviously and is superior to the CBSR method.

\section{Experimental Verification}

To prove the validity of the BCPSR method, the new method is carried over into the fault characteristic frequency extraction of a slight damage inner race of bearing. To further analyze the powerful effect of the new method, the experimental data of missing tooth and broken tooth of planetary gearbox are used to prove the validation of the new method.

4.1. Experimental Verification of the Bearing. Rolling bearing is the key component of rotating equipment, and the working state of the rolling bearing is bound up with the operation reliability of the rotating machinery. However, the rolling bearing is one of the most easily damaged parts in the machine. In this case, the extraction of the slight damage inner race of bearing signal is used to confirm the effectiveness of the BCPSR method. Mechanical equipment failure comprehensive test bench was used in the experiment, as shown in Figure 7. ER-10k rolling bearing is used as the fault bearing with its size $D=33.5, Z=8, d=7.9395$, $\alpha=0^{\circ}$, (pitch circle diameter of bearing is $33.5 \mathrm{~mm}$, the amount of rolling elements is 8 , the diameter of rolling elements is $7.9395 \mathrm{~mm}$, and contacting angle of bearing load is 0). The sampling frequency is $2560 \mathrm{~Hz}$. According to the vibration theory analysis, the characteristic frequency of the inner race of bearing is $212.85 \mathrm{~Hz}$, and the time-domain waveform, power spectrum, and envelope spectrum of the original signal are shown in Figure 8. It can be concluded that the target frequency cannot be extracted in the power spectrum and envelope spectrum because the characteristic frequency signal is polluted by powerful external interference signals. Hence, the BCPSR method is used to extract the target frequency of the inner race of bearing. The time-domain waveform and power spectrum of the output waveform are shown in Figures 9(a) and 9(b). In Figure 9(b), the characteristic frequency spectrum peak is $213.12 \mathrm{~Hz}$, 


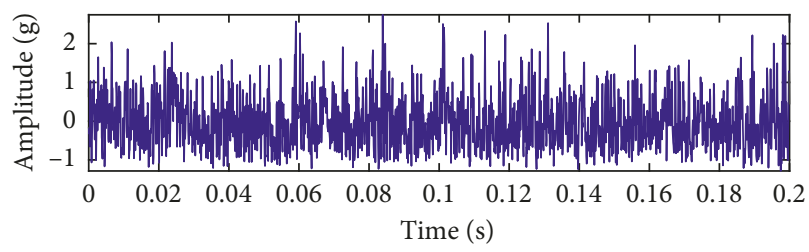

(a)

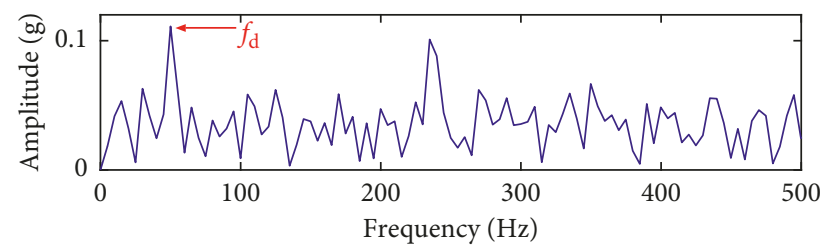

(b)

Figure 5: (a) Time-domain waveform of envelope signal and (b) corresponding spectrum.

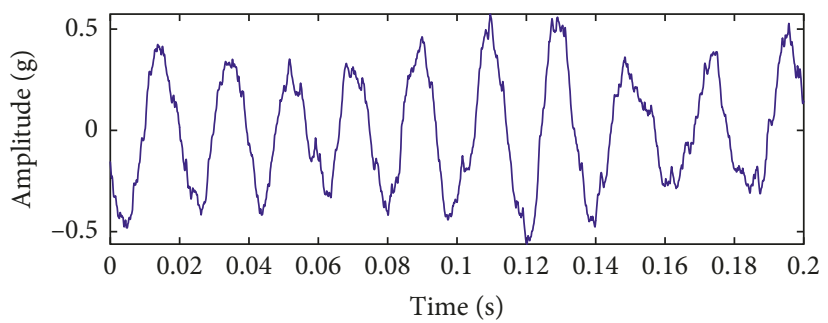

(a)

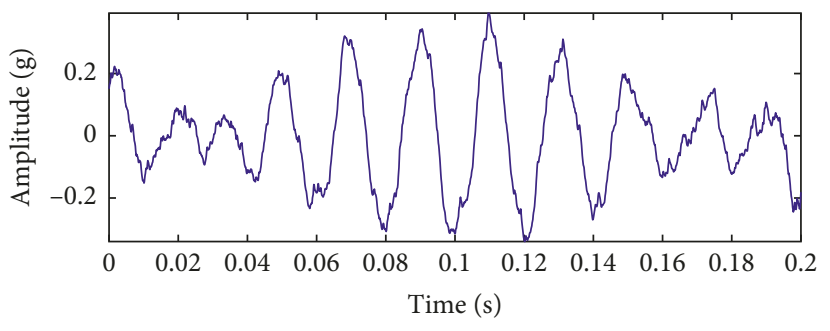

(c)

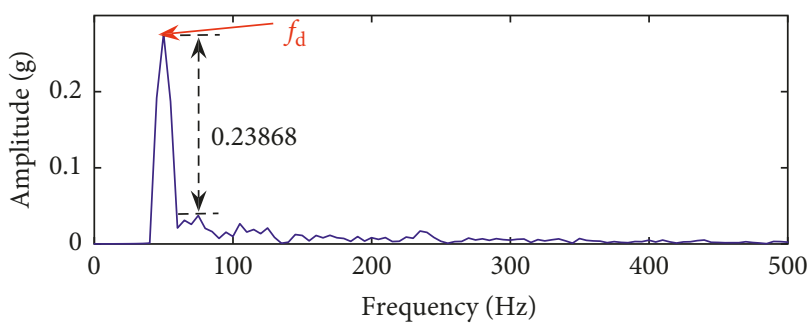

(b)

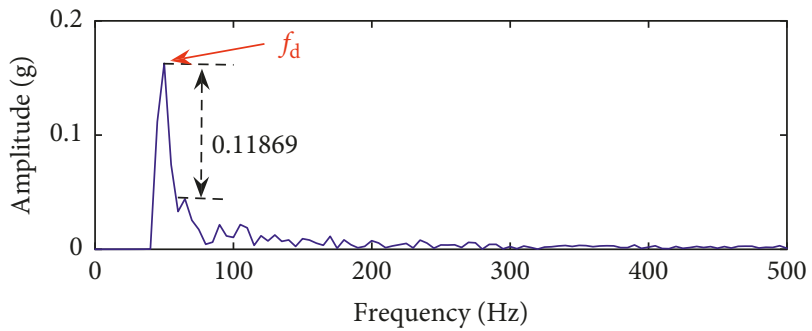

(d)

FIgURE 6: (a) Optimal output signal using the proposed BCPSR method and (b) corresponding power spectrum; (c) optimal output signal using the CBSR method and (d) corresponding power spectrum.

which is much higher than the surrounding noise, and it is closer to the theoretical value $212.85 \mathrm{~Hz}$. Hence, we can draw that the target frequency of the bearing inner race has been identified by proposed method. To indicate the better effect of the BCPSR method, the collected signals are applied to the CBSR method, and the time-domain waveform and power spectrum of the output waveform are shown in Figures 9 (c) and 9(d). The target frequency of the inner race of bearing is also extracted by the CBSR method, but it can be clearly found that the BCPSR method has better effect than the CBSR method. The spectrum peaks of two methods are 0.2051 and 0.1289 , respectively. Here, the difference between spectrum peak of the characteristic frequency and the maximum surrounding noise is defined as the recognition degree. The recognition degree of the BCPSR method and the CBSR method is 0.10913 and 0.05183 , respectively. The output SNR is $22.68 \mathrm{~dB}$ and $21.22 \mathrm{~dB}$, respectively. From the above results, it can be concluded that the BCPSR method has higher spectral peak, recognition degree, and output SNR than the CBSR method in the fault diagnosis experiment of the inner race of bearing. So, it can judged that the proposed BCPSR method has a better effect.

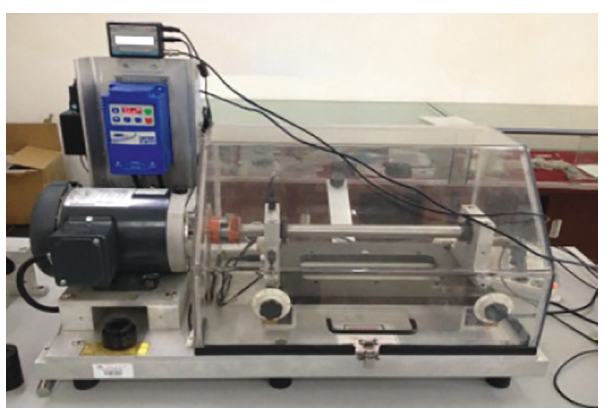

Figure 7: Mechanical equipment failure comprehensive test bench.

4.2. Experimental Verification of the Planetary Gearbox. Comparing with ordinary fixed-axis gearbox, planetary gearbox structure is more complex and has unique operation mode and common complex dynamic load forces. Therefore, the fault diagnosis problem of planetary gearbox should be taken seriously. In this case, the fault extraction experiment on missing tooth and broken tooth of the planetary gearbox is used to prove the correctness of the BCPSR method. The test platform of planetary gearbox is used in the experiment. The three-dimensional model and physical model of the 


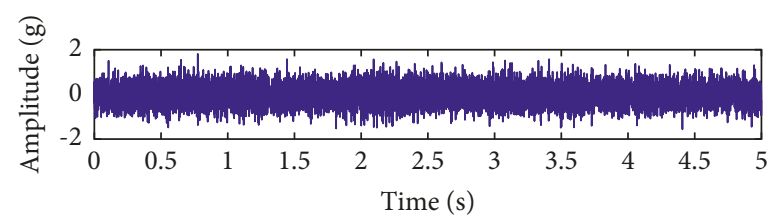

(a)

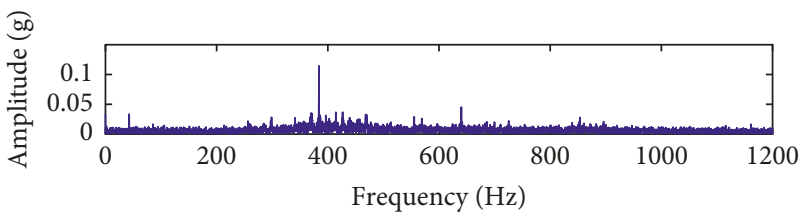

(b)

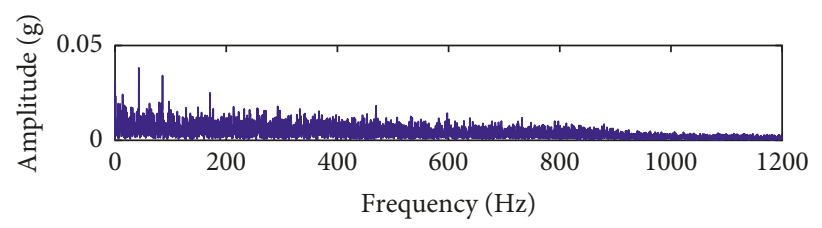

(c)

FIGURE 8: Vibration signal and the spectrum of slight damage inner race of bearing: (a) time-domain waveform, (b) power spectrum, and (c) envelope spectrum.

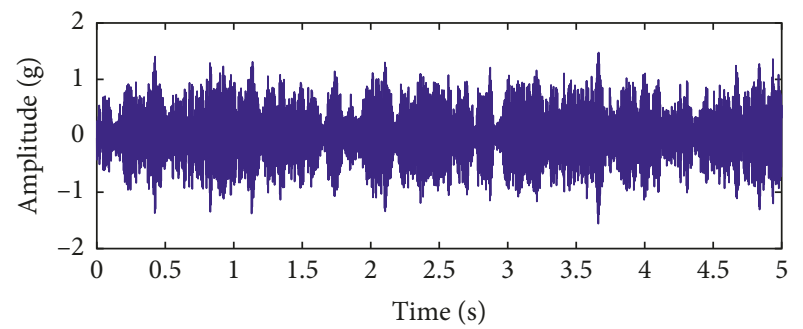

(a)

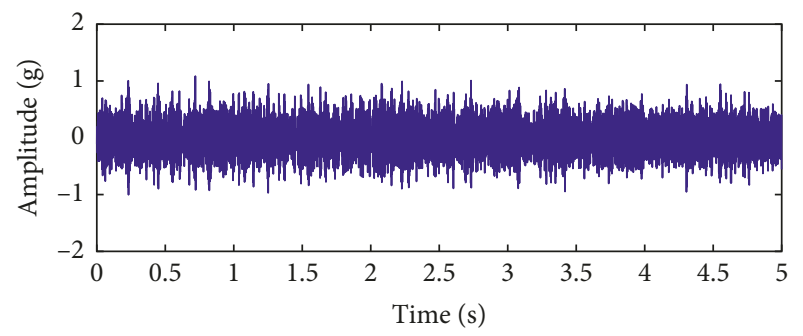

(c)

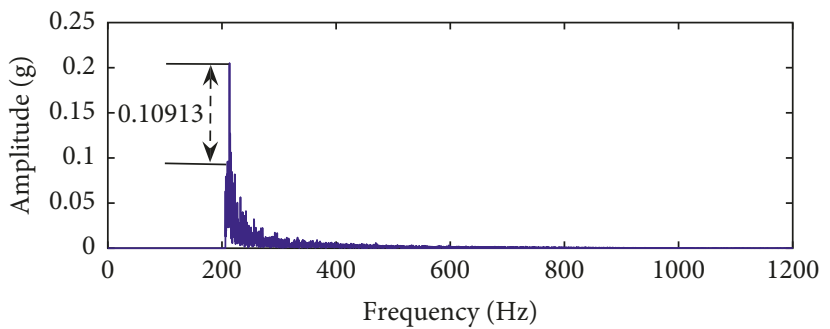

(b)

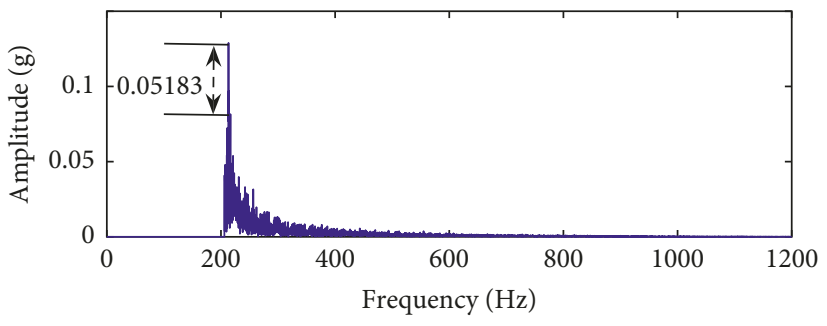

(d)

FIGURE 9: A slight damage inner race of bearing: (a) optimal output signal using the proposed BCPSR method and (b) corresponding power spectrum; (c) optimal output signal using the CBSR method and (d) corresponding power spectrum.

system bench are shown in Figures 10(a) and 10(b). The firststage sun gear is the tested gear. Parameters of planetary gearbox are shown in Table 1. The physical model of the fault gear is shown in Figures 11(a) and 11(b). In order to simulate the heavy load operation of the planetary gearbox, the brake set voltage and current are $1.2 \mathrm{~V}$ and $1.1 \mathrm{~A}$, respectively. According to the vibration theory, the characteristic frequency of the first-stage planetary gearbox is calculated and shown in Table 2.

The time-domain, power spectrum, and envelope spectrum of the collected missing tooth and broken tooth signals are shown in Figure 12, respectively. Due to the complex operation of the planetary gearbox, heavy loads and heavy noises, the meshing frequency, and sidebands frequency are not recognized by noise interference. Therefore, so as to compare the BCPSR method and the CBSR method, the two methods are carried over into identify the fault characteristic frequency. The missing tooth of planetary gearbox optimal output signal using the proposed BCPSR method and CBSR method is displayed in Figure 13. In Figure 13(b), the spectral peak of meshing frequency and sidebands frequency is much higher than the surrounding noise by the BCPSR method. The distance between the meshing frequency and the sidebands frequency is 9.8 and 9.7, respectively, which is very closely to the theoretical value 10. The above analysis shows that the sun gear of the firststage planetary gearbox was a failure. In Figure 13(d) with the CBSR method, the distance between the meshing frequency and the sidebands frequency is 9.6 and 9.4, which is closer to the theoretical value 10 . Therefore, it can be concluded that the first-stage sun gear has been destroyed, but the spectral peaks of the meshing frequency and the sideband frequency are lower than those of the BCPSR method. Similarly, the fault characteristic frequency 


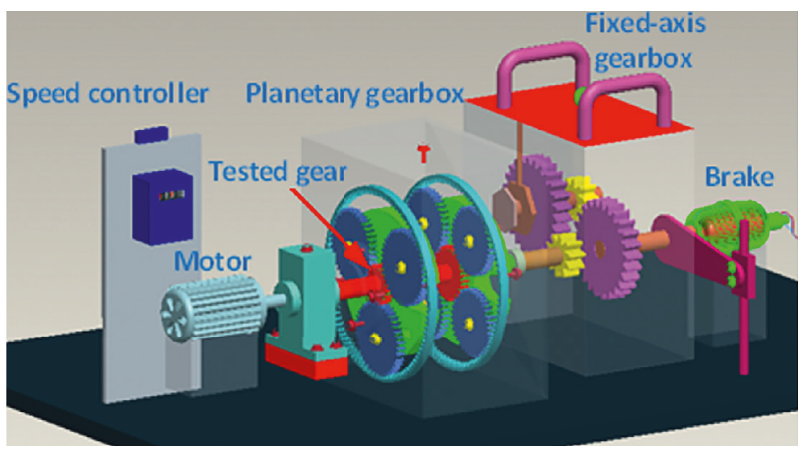

(a)

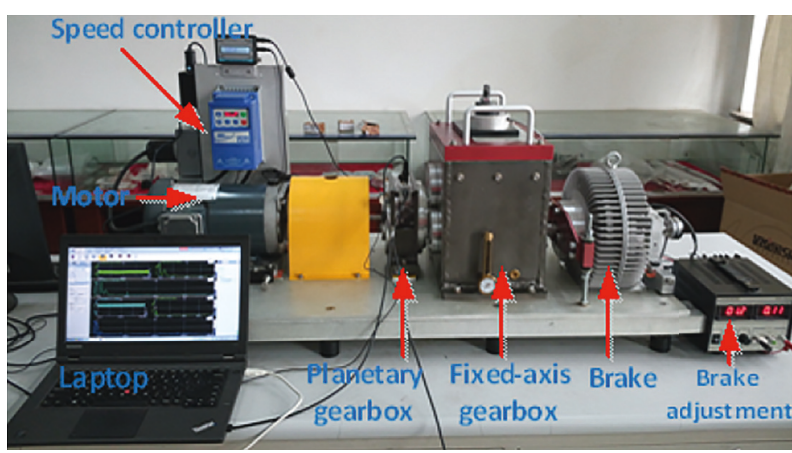

(b)

FIGURE 10: The experimental platform of planetary gearbox: (a) three-dimensional model and (b) physical map.

TABLE 1: Parameters of planetary gearbox.

\begin{tabular}{lcccc}
\hline Forms of gear & Sun gear & Planet gear & Gear ring & Number of planetary gear \\
\hline Teeth number of stage 1 & 20 & 40 & 100 & 3 \\
Teeth number of stage 2 & 28 & 36 & 100 & 4 \\
\hline
\end{tabular}

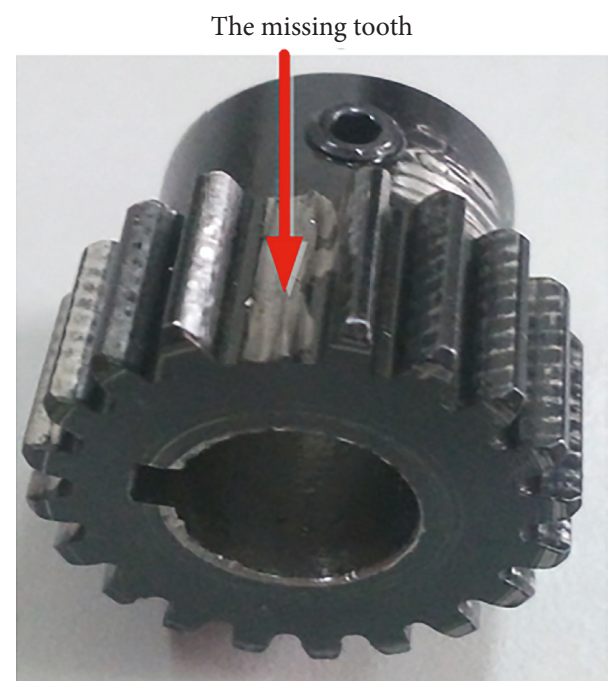

(a)

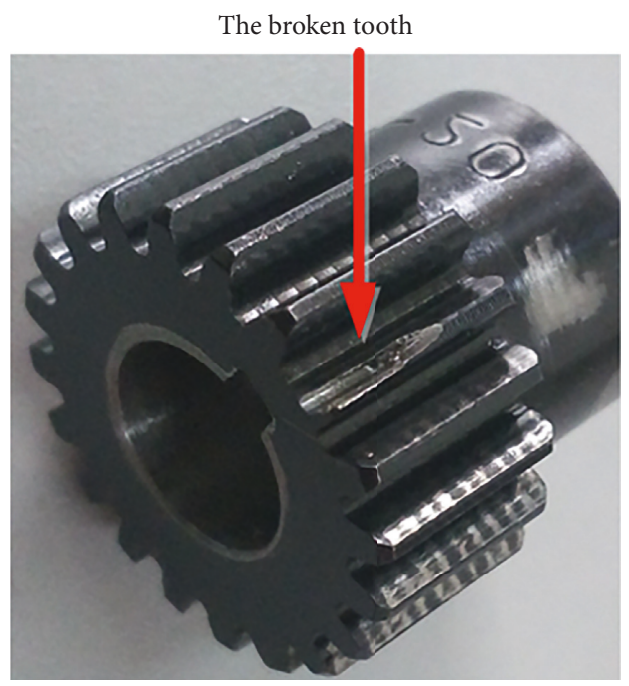

(b)

Figure 11: The physical map of experimental gears: (a) missing tooth and (b) broken tooth.

TABLE 2: Motor speed and the characteristic frequency of the first-stage planetary gearbox.

\begin{tabular}{lcccc}
\hline $\begin{array}{l}\text { Motor } \\
\text { speed }\end{array}$ & $\begin{array}{c}\text { Rotating frequency of sun gear } \\
\left(f_{\text {sidebands }}\right)\end{array}$ & $\begin{array}{c}\text { Rotating frequency of planet gear } \\
\left(f_{\mathrm{p}}\right)\end{array}$ & $\begin{array}{c}\text { Rotating frequency of gear } \\
\text { ring }\left(f_{\mathrm{r}}\right)\end{array}$ & $\begin{array}{c}\text { Meshing frequency } \\
\left(f_{\text {mesh }}\right)\end{array}$ \\
\hline $600 \mathrm{r} / \mathrm{min}$ & $10 \mathrm{~Hz}$ & $1.667 \mathrm{~Hz}$ & $0 \mathrm{~Hz}$ & $166.667 \mathrm{~Hz}$ \\
\hline
\end{tabular}

extraction experiment of the broken tooth is applied by the two methods, and the time-domain waveform and power spectrum are shown in Figure 14. In Figure 14(b), the spectral peak of meshing frequency and sidebands frequency is clearly highlighted by BCPSR method. However, the CBSR method can see the obvious meshing frequency and a sideband frequency, and the other sideband frequency is disturbed by the noise, resulting in a very small spectral peak.
In addition, comparing the power spectrum in Figures 13(b) and 14(b), the spectrum peak of meshing frequency and sidebands frequency of the missing tooth is higher than that of the broken tooth. This phenomenon shows that the vibration caused by the missing tooth is higher than that of the broken tooth, which is consistent with the actual situation. The SNR calculated by the two methods is as follows: in the CBSR method, the output SNR of the missing tooth signal is 


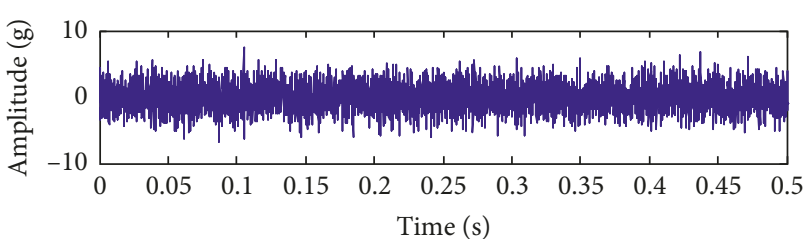

(a)

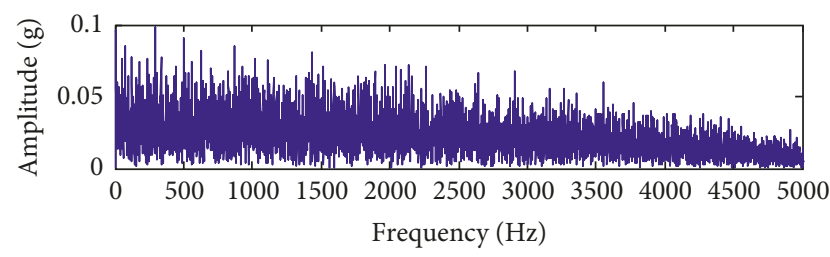

(c)

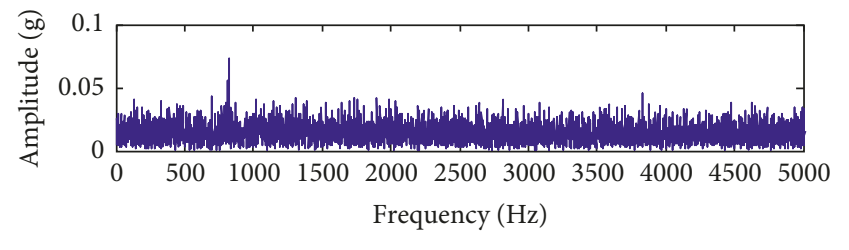

(e)

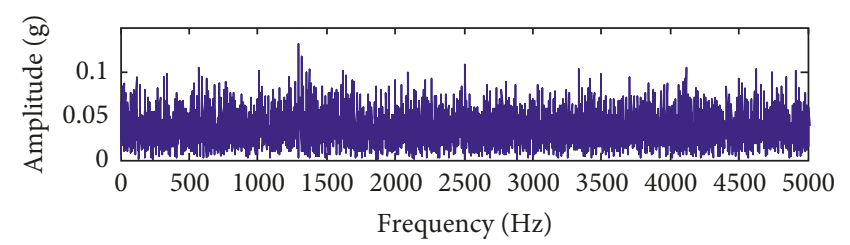

(b)

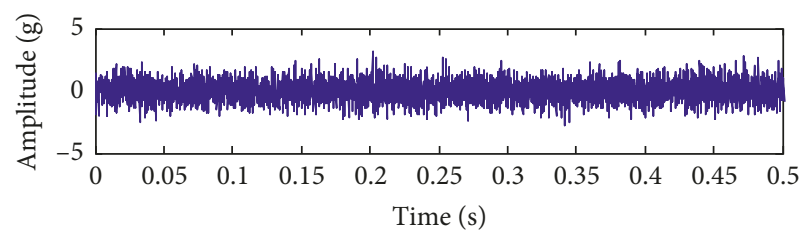

(d)

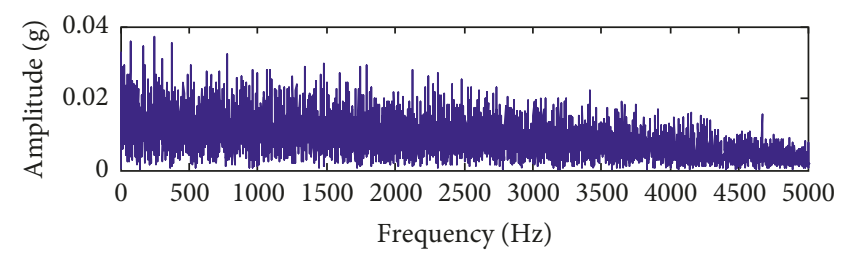

(f)

Figure 12: Vibration signal and the spectrum of the missing tooth: (a) time-domain waveform, (b) power spectrum, and (c) envelope spectrum. Vibration signal and the spectrum of the broken tooth: (d) time-domain waveform, (e) power spectrum, and (f) envelope spectrum.

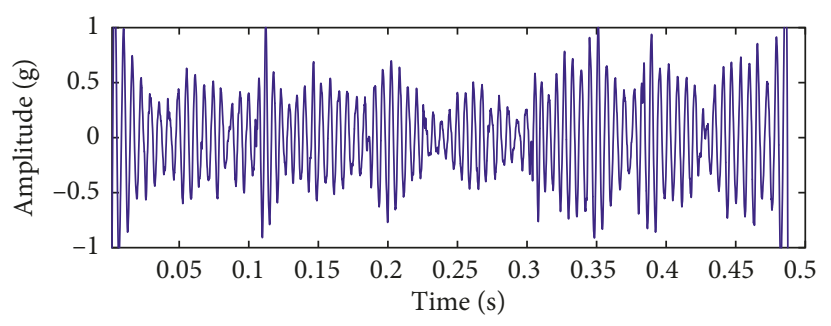

(a)

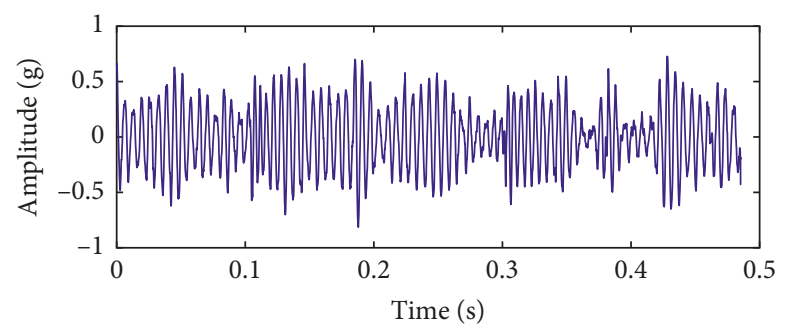

(c)

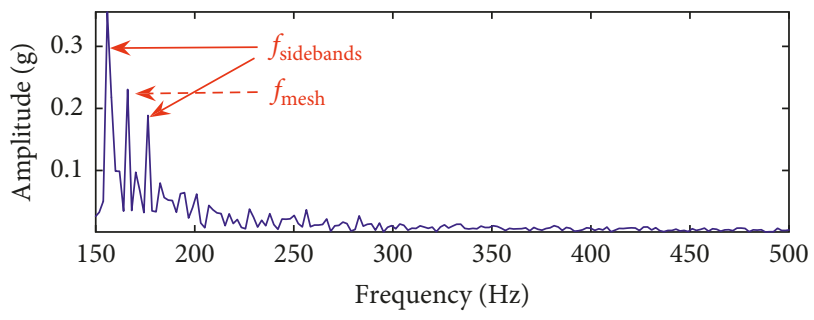

(b)

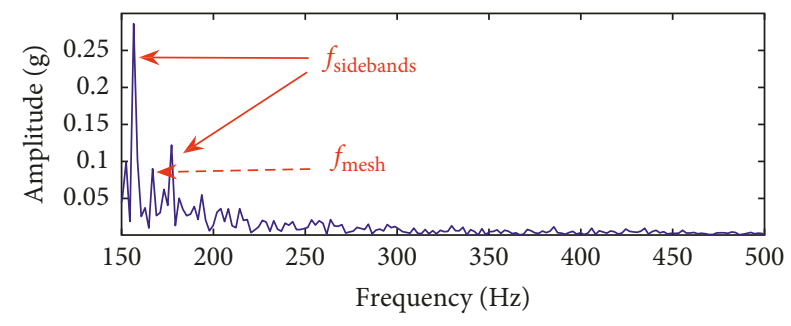

(d)

FIGURE 13: The missing tooth of planetary gearbox: (a) optimal output signal using the proposed BCPSR method and (b) corresponding power spectrum; (c) optimal output signal using the CBSR method and (d) corresponding power spectrum.

23.08 $\mathrm{dB}$ and the output SNR of the broken tooth signal is $21.42 \mathrm{~dB}$. In the BCPSR method, the output SNR of the missing tooth signal is $25.06 \mathrm{~dB}$, and the SNR of the broken tooth output signal is $22.18 \mathrm{~dB}$. Therefore, it can be concluded that the BCPSR method is validated in the fault extraction experiment of the planetary gearbox. Comparing the BCPSR method with the CBSR method, the BCPSR method has higher spectrum peak and better recognition degree and better weak fault signal enhancement capability.

\section{Conclusions}

As the system parameters of the CBSR method are optimized, it is difficult to obtain the perfect potential model structure due to the simultaneous change of the barrier height and width, which hinders the enhancement of the weak fault characteristic frequency and reduces the output SNR. In order to overcome this shortcoming, a new method for the BCPSR is proposed. The following conclusions are drawn: 


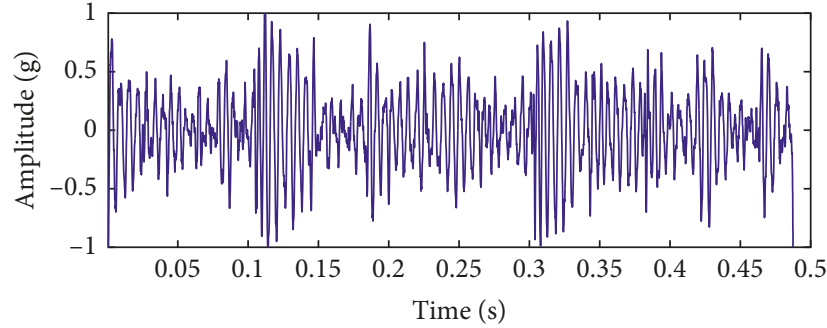

(a)

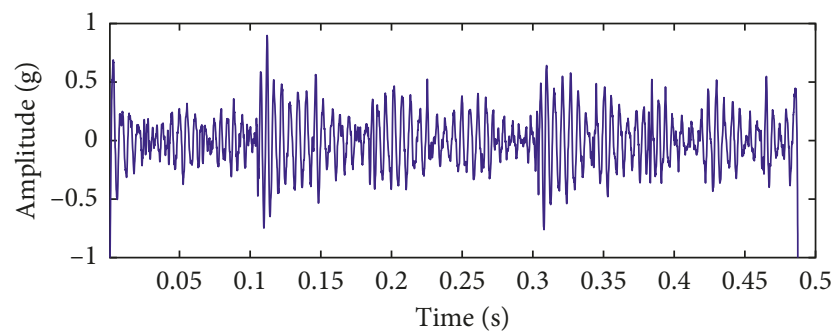

(c)

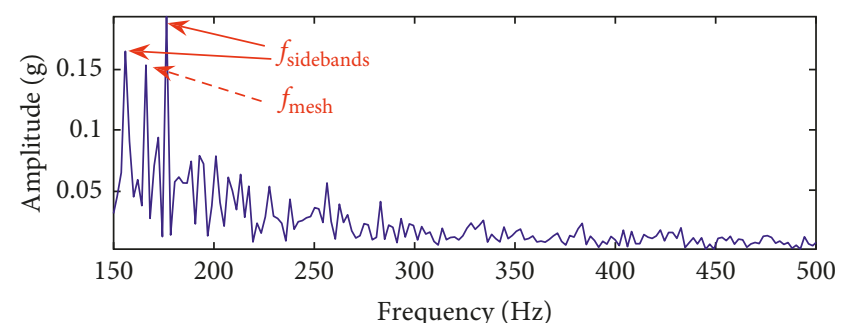

(b)

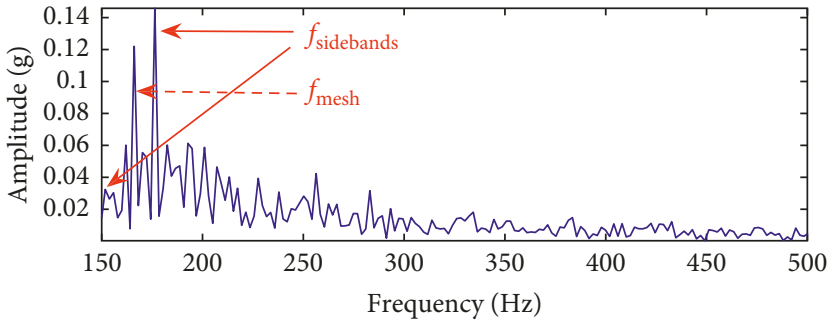

(d)

FIGURE 14: The broken tooth of planetary gearbox: (a) optimal output signal using the proposed BCPSR method and (b) corresponding power spectrum; (c) optimal output signal using the CBSR method and (d) corresponding power spectrum.

(1) The BCPSR system model is analyzed and its potential function and structural characteristics are described. Compared with the CBSR system, the BCPSR system parameters can not only be independently adjusted for the local shape of the potential model but also have a higher theoretical output SNR, which implies that it can better detect and extract the weak signal in the strong background noise.

(2) When the barrier height of the CBSR system potential model is adjusted, the potential width is changed and vice versa. When the potential width and barrier height are changed synchronously, the potential structure will not be perfect. The BCPSR system potential model can make the potential width, barrier height, and wall steepness adjusted independently and simultaneously, so the potential model has better matching parameters.

(3) The proposed BCPSR method is applied to a slight damage inner race of bearing and the missing tooth and broken tooth of the planetary gearbox. The experiment indicates that the BCPSR method not only can extract the weak characteristic frequency but also has better recognition, higher fault characteristic frequency spectrum peak, and better SNR than the CBSR method.

(4) The proposed BCPSR method is based on the overdamped state. The SNR output and the weak fault diagnosis method of the BCPSR on the underdamped state and the factors of affecting the saturation for the CBSR are the focus of further work in the future. In addition, the proposed BCPSR method is applied to the actual engineering verification of the planetary gearbox to further determine the application effect of the method in engineering.

\section{Data Availability}

The data used to support the findings of this study are available from the corresponding author upon request.

\section{Conflicts of Interest}

The authors declare that they have no conflicts of interest.

\section{Acknowledgments}

This research was supported by the National Science Foundation of China (51805275).

\section{References}

[1] P. Goldman and A. Muszynska, "Application of full spectrum to rotating machinery diagnostics," Orbit, vol. 1, pp. 17-21, 1999.

[2] J. Wodecki, P. Stefaniak, J. Obuchowski, A. Wylomanska, and R. Zimroz, "Combination of principal component analysis and time-frequency representations of multichannel vibration data for gearbox fault detection," Journal of Vibroengineering, vol. 18, no. 4, pp. 2167-2175, 2016.

[3] P. D. Samuel and D. J. Pines, "A review of vibration-based techniques for helicopter transmission diagnostics," Journal of Sound and Vibration, vol. 282, no. 1-2, pp. 475-508, 2005.

[4] N. Sawalhi and R. B. Randall, "Vibration response of spalled rolling element bearings: observations simulations and signal processing techniques to track the spall size," Mechanical Systems and Signal Processing, vol. 25, no. 3, pp. 846-870, 2011.

[5] F. B. Duan, F. C. Blondeau, and D. Abbott, "Exploring weakperiodic-signal stochastic resonance in locally optimal processors with a Fisher information metric," Signal Processing, vol. 92, no. 12, pp. 3049-3055, 2012. 
[6] J. L. Chen, Z. P. Li, J. Pan et al., "Wavelet transform based on inner product in fault diagnosis of rotating machinery: a review," Mechanical Systems and Signal Processing, vol. 70-71, pp. 1-35, 2016.

[7] H. R. Cao, F. Fan, K. Zhou, and Z. J. He, "Wheel-bearing fault diagnosis of trains using empirical wavelet transform," Measurement, vol. 82, pp. 439-449, 2016.

[8] Z. X. Li and B. Q. Shi, "Research of fault diagnosis based on sensitive intrinsic mode function selection of EEMD and adaptive stochastic resonance," Shock and Vibration, vol. 2016, Article ID 2841249, 12 pages, 2016.

[9] Z. H. Wu and N. E. Huang, "Ensemble empirical mode decomposition: A noise-assisted data analysis method," Advances in Adaptive Data Analysis, vol. 1, no. 1, pp. 1-41, 2011.

[10] J. S. Smith, "The local mean decomposition and its application to EEG perception data," Journal of the Royal Society Interface, vol. 2, no. 5, pp. 443-454, 2005.

[11] Y. B. Li, M. Q. Xu, R. X. Wang, and W. H. Huang, "A fault diagnosis scheme for rolling bearing based on local mean decomposition and improved multiscale fuzzy entropy," Journal of Sound and Vibration, vol. 360, pp. 277-299, 2016.

[12] Z. J. Qiao and Z. R. Pan, "SVD principle analysis and fault diagnosis for bearings based on the correlation coefficient," Measurement Science and Technology, vol. 26, no. 8, article 085014, 2015.

[13] F. Y. Cong, J. Chen, G. M. Dong, and F. G. Zhao, "Short-time matrix series based singular value decomposition for rolling bearing fault diagnosis," Mechanical Systems and Signal Processing, vol. 34, no. 1-2, pp. 218-230, 2013.

[14] Z. P. Feng and M. Liang, "Complex signal analysis for planetary gearbox fault diagnosis via shift invariant dictionary learning,” Measurement, vol. 90, pp. 382-395, 2016.

[15] Z. J. Qiao, Y. G. Lei, and J. Lin, "Stochastic resonance subject to multiplicative and additive noise: the influence of potential asymmetries," Physical Review E, vol. 94, no. 5, article 052214, 2016.

[16] R. Benzi, A. Sutera, and A. Vulpiana, "The mechanism of stochastic resonance," Journal of Physics A: Mathematical and General, vol. 14, no. 11, pp. L453-L457, 1981.

[17] Y. G. Lei, Z. J. Qiao, and X. Xu, "Weak signal detection based on underdamped multistable stochastic resonance," in Proceedings of IEEE International Instrumentation and Measurement Technology Conference (I2MTC), pp. 1-6, IEEE, Taipei, Taiwan, May 2017.

[18] Y. G. Leng, Y. S. Leng, T. Y. Wang, and Y. Guo, "Numerical analysis and engineering application of large parameter stochastic resonance," Journal of Sound and Vibration, vol. 292, no. 3-5, pp. 788-801, 2006.

[19] J. Y. Tan, X. F. Chen, J. Y. Wang et al., "Study of frequencyshifted and re-scaling stochastic resonance and its application to fault diagnosis," Mechanical Systems and Signal Processing, vol. 23, no. 3, pp. 811-822, 2009.

[20] Z. H. Lai and Y. G. Leng, "Weak-signal detection based on the stochastic resonance of bistable duffing oscillator and its application in incipient fault diagnosis," Mechanical Systems and Signal Processing, vol. 81, pp. 60-74, 2016.

[21] Y. G. Lei, Z. G. Qiao, and X. Xu, "An underdamped stochastic resonance method with stable-state matching for incipient fault diagnosis of rolling element bearings," Mechanical Systems and Signal Processing, vol. 94, pp. 148-164, 2017.

[22] S. L. Lu, Q. B. He, H. B. Zhang, and F. R. Kong, "Rotating machine fault diagnosis through enhanced stochastic resonance by full-wave signal construction," Mechanical Systems and Signal Processing, vol. 85, pp. 82-97, 2017.
[23] X. L. Liu, J. H. Yang, H. G. Liu, G. Cheng, X. H. Chen, and $\mathrm{D}$. Xu, "Optimizing the adaptive stochastic resonance and its application in fault diagnosis," Fluctuation and Noise Letters, vol. 14, no. 4, article 1550038, 2015.

[24] R. C. Zheng, K. Nakano, H. G. Hu, D. X. Su, and M. P. Cartmell, "An application of stochastic resonance for energy harvesting in a bistable vibrating system," Journal of Sound and Vibration, vol. 333, no. 12, pp. 2568-2587, 2014.

[25] X. X. Ding, Q. B. He, and N. W. Luo, "A fusion feature and its improvement based on locality preserving projections for rolling element bearing fault classification," Journal of Sound and Vibration, vol. 335, pp. 367-383, 2015.

[26] D. Y. Han, P. li, S. J. An, and P. M. Shi, "Multi-frequency weak signal detection based on wavelet transform and parameter compensation band-pass multi-stable stochastic resonance," Mechanical Systems and Signal Processing, vol. 70-71, pp. 995-1010, 2016.

[27] P. M. Shi, Q. Li, and D. Y. Han, "Stochastic resonance in a new asymmetric bistable system driven by unrelated multiplicative and additive noise," Chinese Journal of Physics, vol. 54, no. 4, pp. 526-532, 2016.

[28] G. Y. Li, J. M. Li, S. Wang, and X. F. Chen, "Quantitative evaluation on the performance and feature enhancement of stochastic resonance for bearing fault diagnosis," Mechanical Systems and Signal Processing, vol. 81, pp. 108-125, 2016.

[29] F. B. Duan, F. Chapeau-Blondeau, and D. Abbott, "Encoding efficiency of suprathreshold stochastic resonance on stimulusspecific information," Physics Letters A, vol. 380, no. 1-2, pp. 33-39, 2016.

[30] Z. X. Li and B. Q. Shi, "An adaptive stochastic resonance method for weak fault characteristic extraction in planetary gearbox," Journal of Vibroengineering, vol. 19, no. 3, pp. 1782-1792, 2017.

[31] Z. X. Li and B. Q. Shi, "Extracting weak fault characteristics with adaptive singular value decomposition and stochastic resonance," Transactions of the Chinese Society of Agricultural Engineering, vol. 11, no. 33, pp. 60-68, 2017.

[32] S. L. Lu, Q. B. He, and F. R. Kong, "Stochastic resonance with Woods-Saxon potential for rolling element bearing fault diagnosis," Mechanical Systems and Signal Processing, vol. 45, no. 2, pp. 488-503, 2014.

[33] Z. J. Qiao, Y. G. Lei, J. Lin, and F. Jia, "An adaptive unsaturated bistable stochastic resonance method and its application in mechanical fault diagnosis," Mechanical Systems and Signal Processing, vol. 84, pp. 731-746, 2017. 


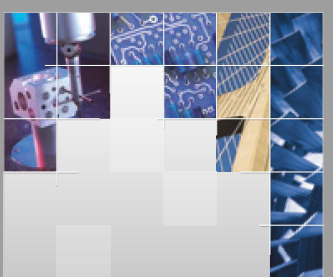

\section{Enfincering}
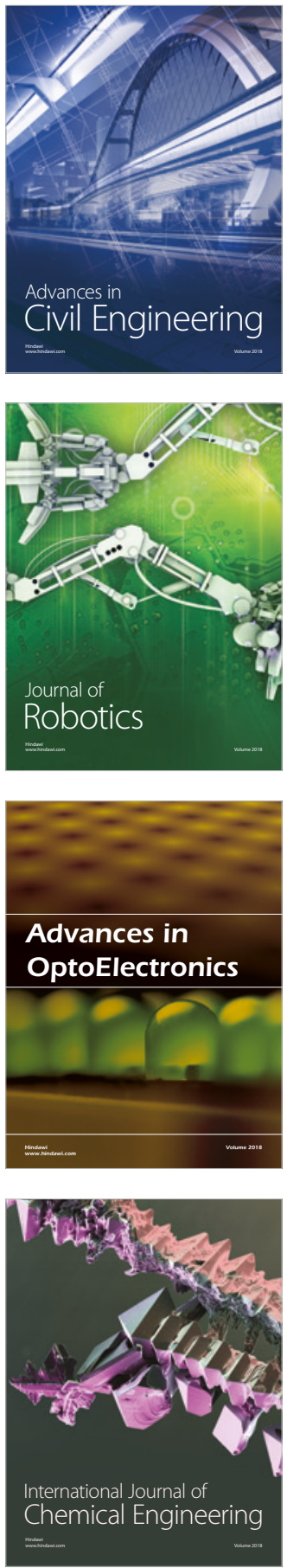

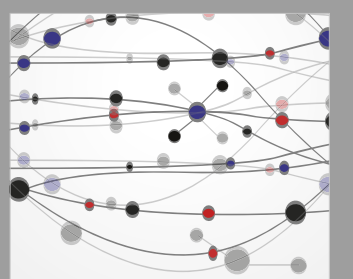

\section{Rotating \\ Machinery}

The Scientific World Journal

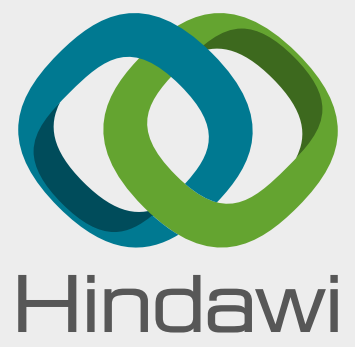

Submit your manuscripts at

www.hindawi.com
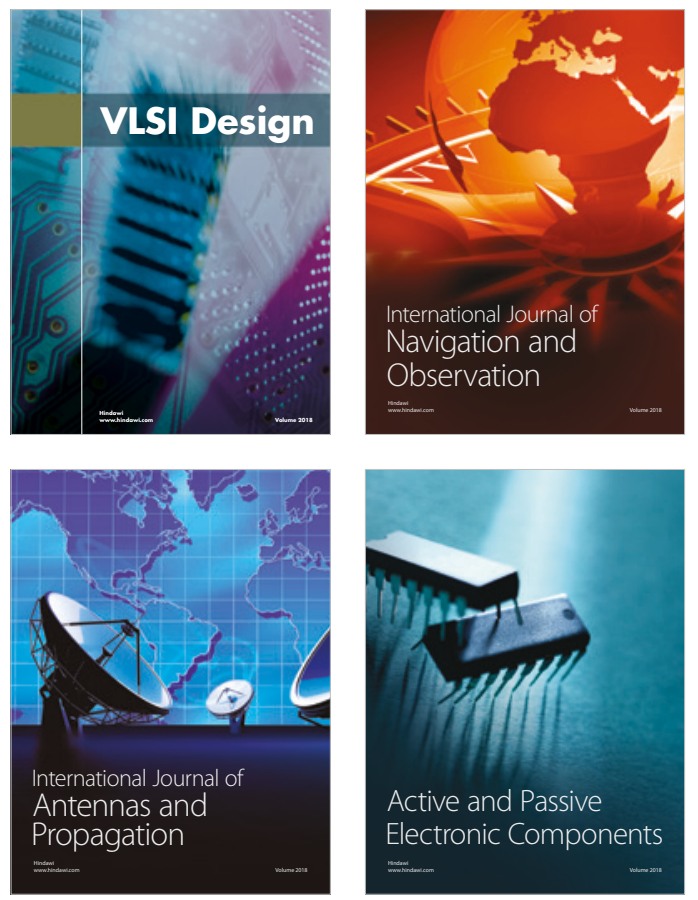
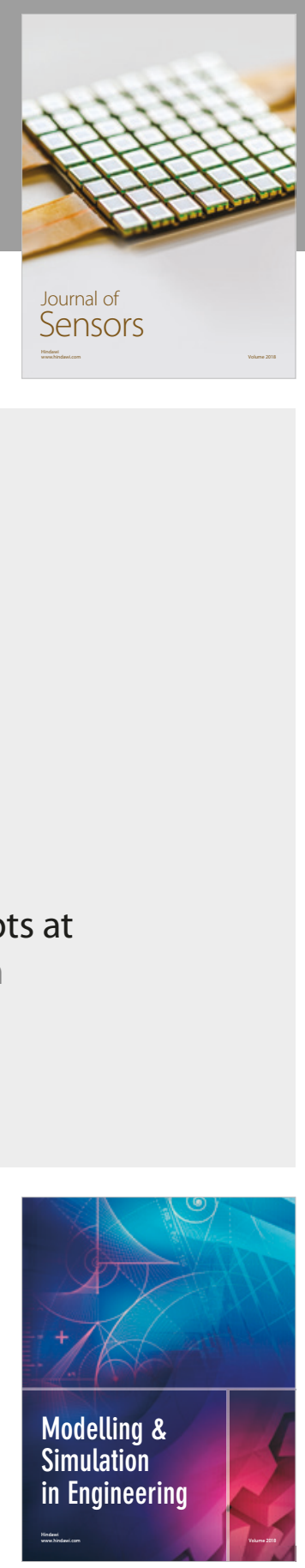

\section{Advances \\ Multimedia}
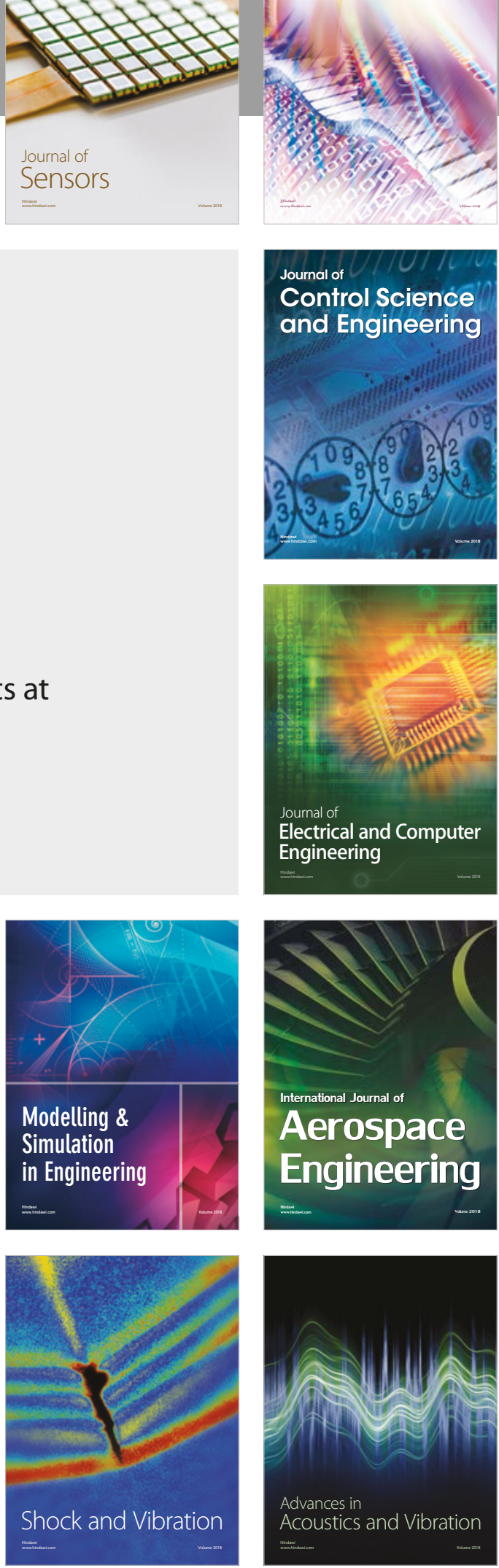